\title{
Sistema móvil para la oferta de recorridos con rutas fijas
}

Fecha de recepción: 26-11-2020 • Fecha de aceptación: 10-01-2021 • Fecha de publicación:10-02-2021

\author{
Christian Andrés Artieda Guachamin ${ }^{1}$ \\ Megadatos - Netlife, Ecuador \\ kristian.ldu@gmail.com \\ https://orcid.org/0000-0001-5500-2511 \\ Juan Sebastián Reza Morales ${ }^{2}$ \\ Tata Consultancy Services, Ecuador \\ jsrezam@gmail.com \\ https://orcid.org/0000-0002-8318-3695 \\ Henry Daniel Rojas Cisneros ${ }^{3}$ \\ Biometrika Cloud Services, Ecuador \\ henry.red1@hotmail.com \\ https://orcid.org/0000-0001-8157-760X
}

\section{RESUMEN}

El presente trabajo busca generar una automatización mediante una aplicación móvil con la cual los usuarios que cuenten con un vehículo, ofrezcan a las personas una opción de viaje a sus destinos, basada en su ruta diaria y a un precio accesible. La idea surge de la necesidad de que los usuarios conductores puedan tener un ingreso extra llevando pasajeros dentro de su recorrido, y a su vez, los pasajeros puedan contar con una aplicación alternativa más económica que les permita llegar más rápido a sus destinos finales. Se utilizan herramientas que son tendencia actualmente, como Firebase para la gestión de datos, Xamarin como principal en cuanto al desarrollo, debido a su gran ventaja de reutilizar el mismo código para las plataformas de Android y IOS, como complemento se utilizarán las APIs de Facebook y Google para brindar al beneficiario una alternativa de inicio de sesión, y Google Maps para el despliegue de rutas en un mapa. Los resultados evidencian que el sistema será una gran opción al momento de gestionar la rutas. 


\section{PALABRAS CLAVE: APIs, xamarin, android, ios, framework.}

\section{ABSTRACT}

This work seeks to generate an automation through a mobile application with which users with a vehicle, offer people a travel option to their destinations, based on their daily route and at an affordable price. The idea arises from the need for users to have an extra income by carrying passengers within their route, and in turn, passengers can have an alternative, cheaper application that allows them to reach their final destinations faster. Tools that are currently a trend are used, such as Firebase for data management, Xamarin as the main development tool, due to its great advantage of reusing the same code for the Android and IOS platforms. As a complement, Facebook and Google APIs will be used to provide the beneficiary with a login alternative, and Google Maps for the display of routes on a map. The results show that the system will be a great option when managing the routes. 


\section{Introducción}

En la actualidad, con el surgimiento de la pandemia por COVID-19, que afectó a todo el mundo y obligó el cierre de varias empresas (Weller, 2020), además del aumento del desempleo en todas las ciudades del mundo, ha generado que el uso de la tecnología juegue un papel muy importante y crezca su demanda entre las personas para poder continuar con sus trabajos de forma remota, precautelar la salud de los empleados, comunicarse con sus familiares y facilitar la movilidad (Torres et al., 2020). Al respecto, Reyes Rivera et al. (2020) agregan que el desarrollo tecnológico y el acceso a grandes cantidades de información en la web han alcanzado un nivel sin precedentes en la historia.

En Ecuador operan diferentes empresas que ofrecen el servicio de VTC (Vehiculos de transporte con conductor) como son Uber (Sitio Oficial de Uber, n.d.), Cabify (Cabify, n.d.), InDriver (InDriver, n.d.), entre otros. Estos servicios han crecido por el surguimiento de la pandemia debido a que las personas, al permanecer en sus hogares, utilizaron y utilizan diferentes plataformas para ir a sus trabajos, enviar paquetes o pedir productos alimenticios, con la intención de prevenir el contagio. Cada servicio es ofrecido a travez de aplicaciones móviles que son accesibles para todo usuario que tenga acceso a un dispositivo móvil (Herrera, 2020).

El auge del desarrollo de aplicaciones moviles (Martinez, 2001) ha sido un factor determinante para facilitar ciertas actividades que requerian que la persone realice esto de forma presencial, por citar ciertos ejemplos los autores Burbano Ulloa et al. (2020) realizan un análisis de las aplicaciones móviles en Ecuador y proponen una aplicación movil para el control de mascotas, en cambio Reyes Rivera et al. (2020) desarrollan un sistema móvil de compras en línea, este tipo de aplicaciones también puede influir en la conciencia de las personas, asi lo demuestran Toasa et al. (2017) que crean una aplicación móvil para concientizar a las personas sobre el uso de la energía eléctrica, y para esto utilizan técnicas de gamificación.

Por lo mencionado anteriormente, el objetivo de este trabajo es ofrecer una nueva oportunidad de empleo a los diferentes usuarios que posean un vehículo y a su vez puedan ofrecer sus servicios a personas que puedan necesitarlo, esto mediante el desarrollo de una aplicación móvil que permita a los usuarios ofrecer sus servicios de conducción por medio de recorridos con rutas fijas, para cumplir esto se ha planificado: definir el diseño de interfaces para la aplicación móvil, establecer rutas fijas a través de un mapa que permite una mejor visualización, registrar usuarios utilizando sus perfiles de redes sociales para agilitar su creación, validar la aplicación desarrollada en un ambiente de pruebas simuladas.

\section{Metodología}

En el presente proyecto se utilizó la metodología Scrum (SCRUM - Proyectos Ágiles, n.d.), la cual permite realizar entregas parciales y regulares del producto final, priorizadas por el beneficio que aportan al receptor del proyecto. Algo importante de la metodología scrum es que para organiar las tareas y tiempos se basa en los principios de KANBAN (Lage Junior \& Godinho Filho, 2010). 
En Scrum, un proyecto se ejecuta en ciclos temporales cortos y de duración fija. Cada iteración tiene que proporcionar un resultado completo un incremento de producto final que sea susceptible de ser entregado con el mínimo esfuerzo al cliente cuando lo solicite. Para este proyecto se decidió hacer iteraciones cada semana teniendo reuniones al final de la misma.

\subsection{Requerimientos}

En la siguiente Tabla 1 se visualizarán la lista de requerimientos presentados para este proyecto.

Tabla 1.

Tabla de Requerimientos

\begin{tabular}{|l|l|}
\hline \multirow{2}{*}{} & Tabla de Requerimientos \\
\cline { 2 - 3 } & Requerimientos \\
\hline 1 & Realizar registro de conductores y pasajeros. \\
\hline 2 & Realizar inicio de sesión o registro mediante redes sociales. \\
\hline 3 & Visualizar las posibles rutas que ofertan los diferentes conductores. \\
\hline 4 & Activar de forma manual la oferta de ruta del conductor hacia los pasajeros. \\
\hline 5 & Verificar el historial de los viajes realizados por el pasajero y el conductor. \\
\hline 6 & Realizar un control del viaje al inicio y al final del recorrido. \\
\hline
\end{tabular}

Fuente: elaboración propia

\subsection{Roles e integrantes}

Mientras que en la Tabla 2 se evidencia el equipo encargado del desarrollo de este proyecto.

Tabla 2.

Tabla de Scrum Team

\begin{tabular}{|l|l|l|}
\hline & Scrum Team \\
\hline 1 & Sebastian Reza & $\begin{array}{l}\text { Scrum Master / Development Team } \\
\text { Member }\end{array}$ \\
\hline 2 & Christian Artieda & Development Team Member \\
\hline 3 & Henry Rojas & Development Team Member \\
\hline
\end{tabular}

Fuente: elaboración propia

\subsection{Iteracciones (Sprints)}

Para la construcción se ejecutaron los siguientes 4 sprint con el siguiente detalle: 


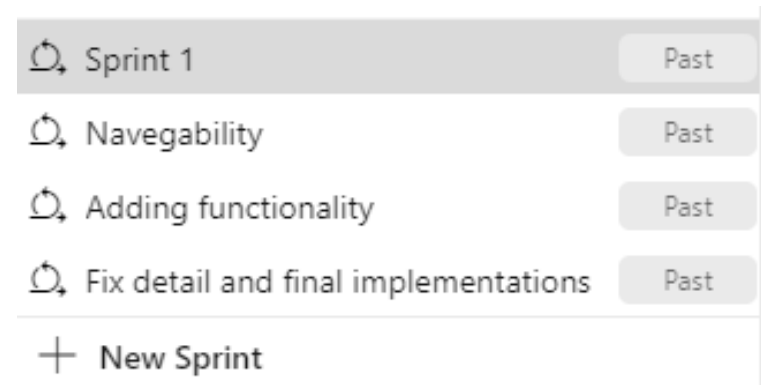

Figura 1. Lista de sprints ejecutados con Azure DevOps.

Fuente: elaboración propia

\section{Primer Sprint}

El primer sprint fue ejecutado del 19 al 25 de octubre de 2020, cubriendo tareas fundamentales para asentar una arquitectura escalable y mantenible, siendo esto un hito fundamental para las siguientes iteraciones donde se fue agregando funcionalidad a la aplicación.

Estas tareas comprenden el diseño e implementación de las clases e interfaces que tienen la responsabilidad de comunicarse con Firebase database para persistir la data en esta plataforma, como punto clave también se creó el repositorio en Github para el manejo y control de código fuente, con esta tarea ejecutada fue posible repartir el trabajo al equipo de desarrollo. Otro punto importante fue la implementación del módulo de inicio de sesión y registro en la aplicación con los patrones GUI que ofrece Xamarin.

En la Figura 2 se detallan las tareas que conformaron el backlog del primer sprint del proyecto.

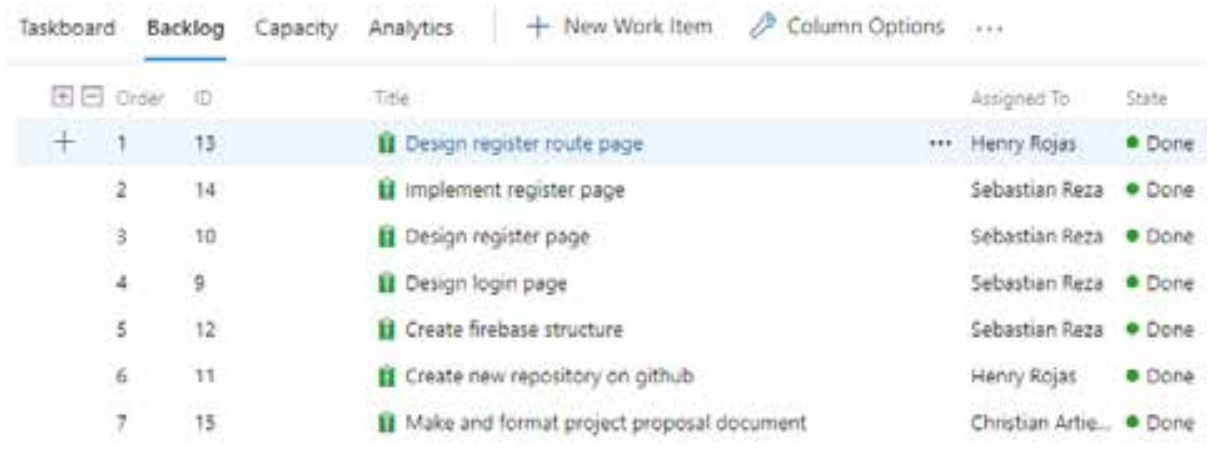

Figura 2. Backlog para el primer sprint en Azure DevOps.

Fuente: elaboración propia

\section{Segundo Sprint}

El segundo sprint fue ejecutado del 26 de octubre al 1 de noviembre de 2020, el objetivo principal de esta iteración fue trabajar en la navegabilidad fundamental visionada para cumplir con el propósito del negocio que plantea la aplicación, lo cual involucró el diseño de las pantallas para 
la selección de la ruta del conductor y las pantallas para la selección de los viajes ofertados para el pasajero, consecuentemente también se diseñaron las pantallas para que el usuario de la aplicación, sea pasajero o conductor, pueda observar su historial de viajes realizados en la aplicación.

Otro reto para este sprint, fue la persistencia de los viajes ofertados en Firebase database, tomando en cuenta todas las validaciones necesarias para que la información se almacene de forma íntegra y consistente en tiempo real.

Por último, para este ciclo se llevó a cabo la implementación de la autenticación con las APIs de Facebook y Google.

En la Figura 3 se pueden ver las tareas que conformaron el backlog del segundo sprint del proyecto.

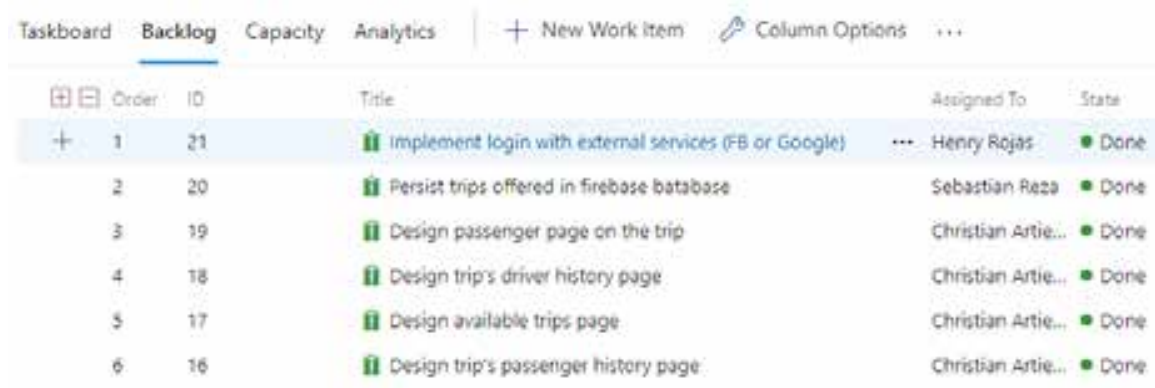

Figura 3. Backlog para el segundo sprint en Azure DevOps.

Fuente: elaboración propia

\section{Tercer Sprint}

El tercer sprint fue ejecutado del 4 al 8 de noviembre del 2020, siendo una iteración crítica para concretar el core de la aplicación. En este sprint se implementó el manejo de roles de pasajeros y conductores.

Terminado el manejo de roles, se finalizó con todas las funcionalidades tanto del conductor, como del pasajero, estas se establecieron como la oferta de las ruta fijas, manejo de los pasajeros propuestos en el viaje e inicio y finalización del viaje.

En la Figura 4 se evidencian las tareas que conformaron el backlog del tercer sprint del proyecto. 


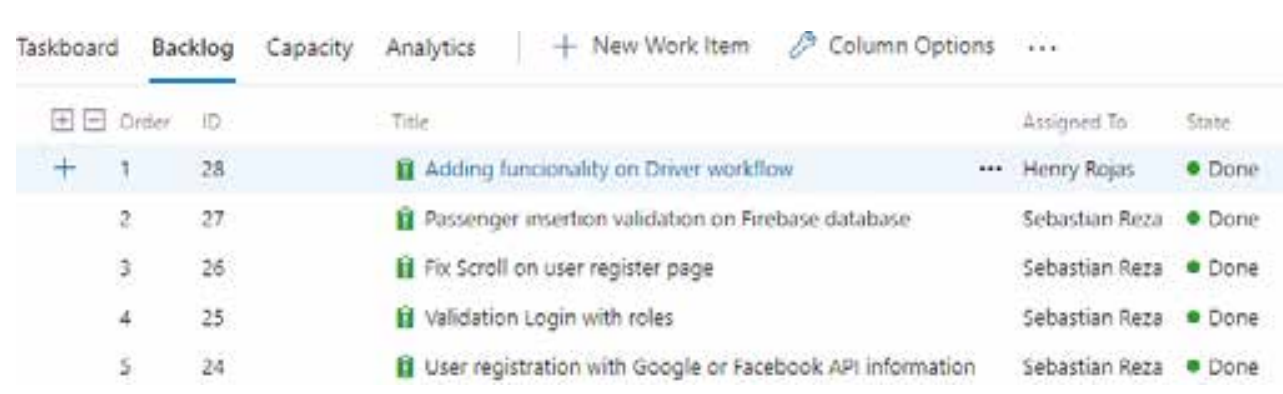

Figura 4. Backlog para el tercer sprint en Azure DevOps.

Fuente: elaboración propia

\section{Cuarto Sprint}

El cuarto y último sprint fue ejecutado del 12 al 18 de noviembre de 2020, este último ciclo se concretó en la eliminación de diferentes bugs encontrados en las pruebas internas y en implementar funcionalidades finales como la implementación y diseño de pantallas para la consulta del perfil y viajes realizados por pasajeros y conductores.

El principal objetivo que se alcanzó en este último sprint fue la sincronización del ingreso o salida de un pasajero en los viajes, al igual que la actualización de la lista de viajes disponibles para el pasajero, finalizando de igual manera con la sincronización entre el conductor y pasajero al momento de culminar o comenzar un viaje.

En la Figura 5 se detallan las tareas que conformaron el backlog del cuarto sprint del proyecto.

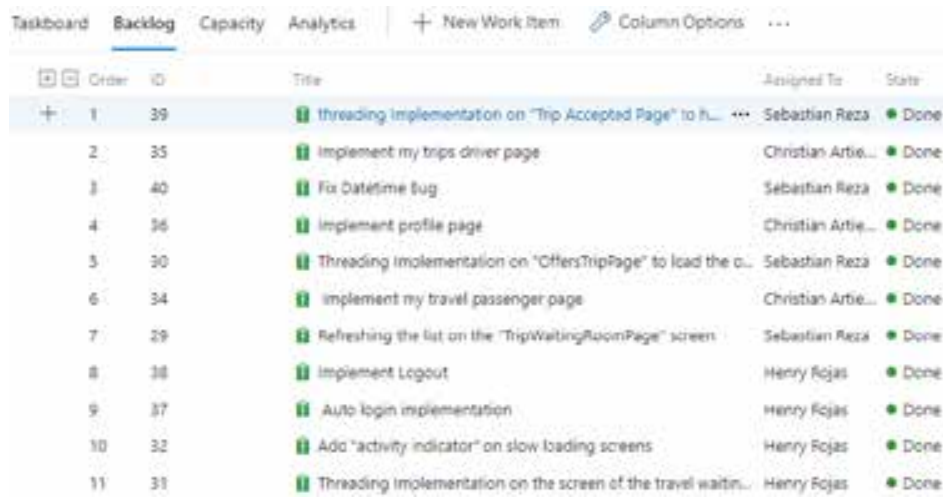

Figura 5. Backlog para el cuarto sprint en Azure DevOps.

Fuente: elaboración propia

\subsection{Herramientas de desarrollo}

En este proyecto se utilizaron las herramientas de desarrollo de Xamarin (Xamarin I Open-Source Mobile App Platform for .NET, n.d.), en el IDE Visual Studio (IDE de Visual Studio, Editor de Código, Azure DevOps y App Center - Visual Studio, n.d.) que va a permitir realizar la codificación, además de realizar las diferentes pruebas en dispositivos móviles. 
Previo al inicio del desarrollo se debe realizar la creación y configuración de las diferentes APIs con las que el proyecto va a funcionar. Estas son:

- Creación de Proyecto Firebase.

- Creación de API Key para mapas de Google.

- Creación de Proyecto Facebook.

En las Figuras 6, 7, 8, 9 y 10 se puede visualizar el proceso de creación del proyecto en Firebase para la administración de usuarios y el almacenamiento de información.

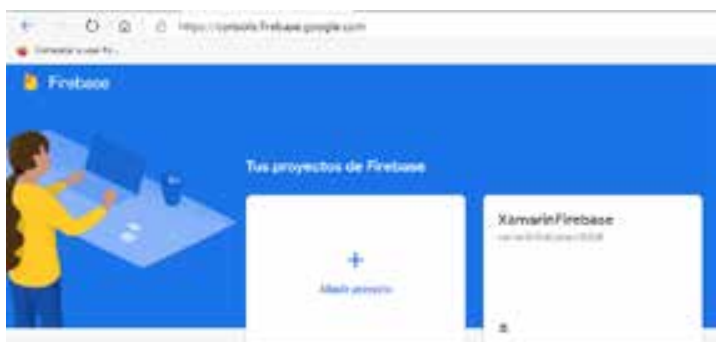

Figura 6. Consola de administración de proyectos

Fuente: elaboración propia

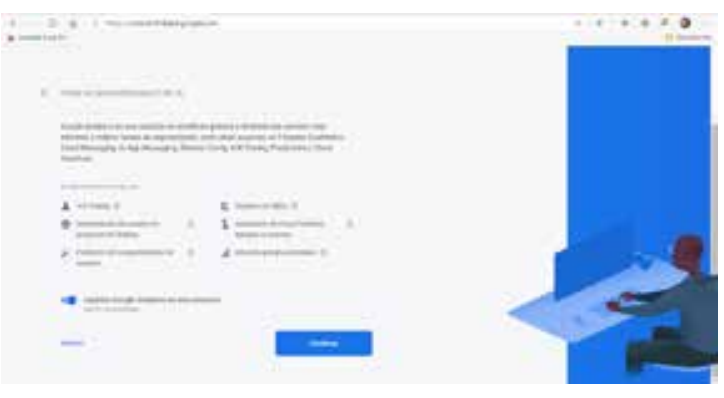

Figura 8. Creación de proyecto Firebase Fuente: elaboración propia

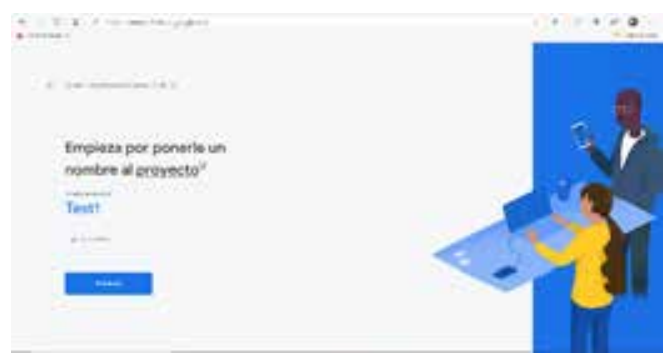

Figura 7. Creación de proyecto en Firebase Fuente: elaboración propia

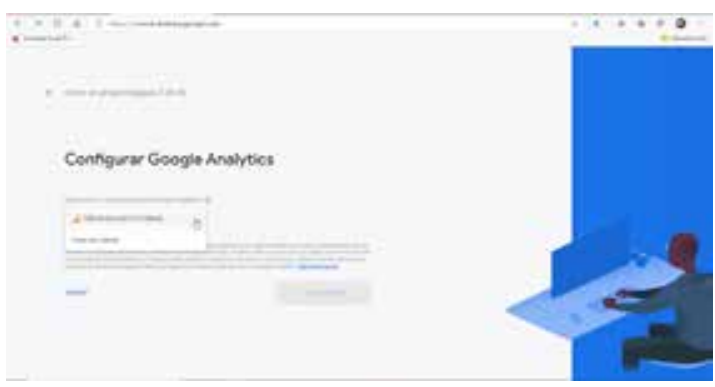

Figura 9. Creación de proyecto Firebase Fuente: elaboración propia 


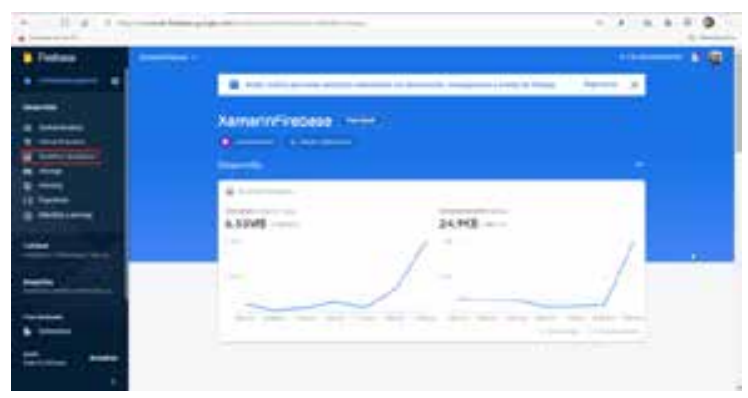

Figura 10. Consola de administración del proyecto creado

Fuente: elaboración propia

En las Figuras 11, 12 y 13 se puede visualizar la generación del API Key para el manejo de mapas de Google para la plataforma Android, asociado al proyecto Firebase con el cual va a funcionar la aplicación.

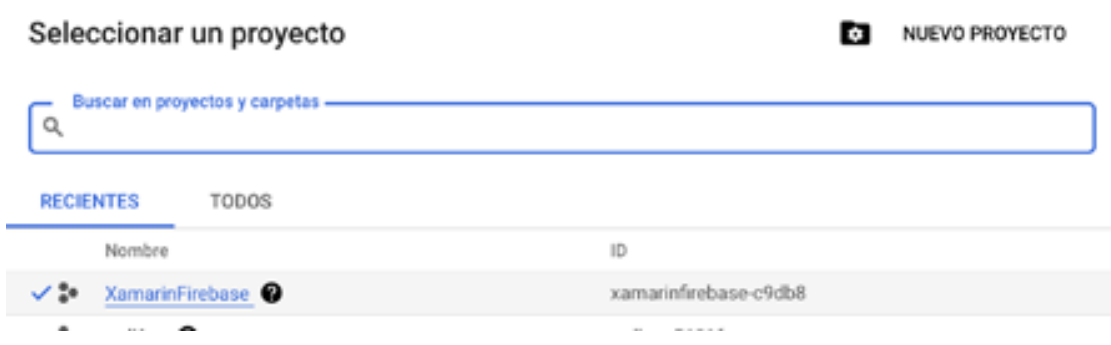

Figura 11. Selección de proyecto de Firebase

Fuente: elaboración propia

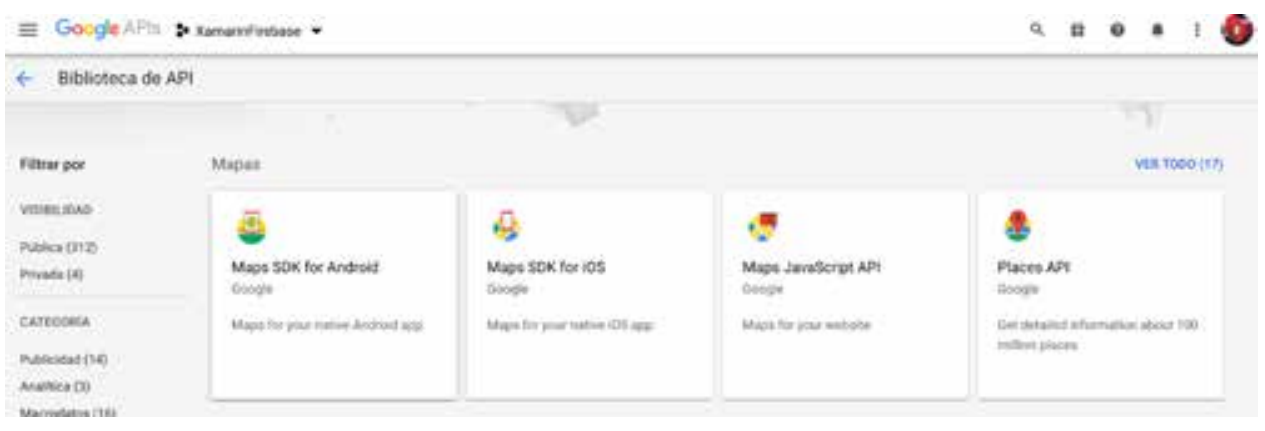

Figura 12. Habilitación de API de mapas para Android

Fuente: elaboración propia 


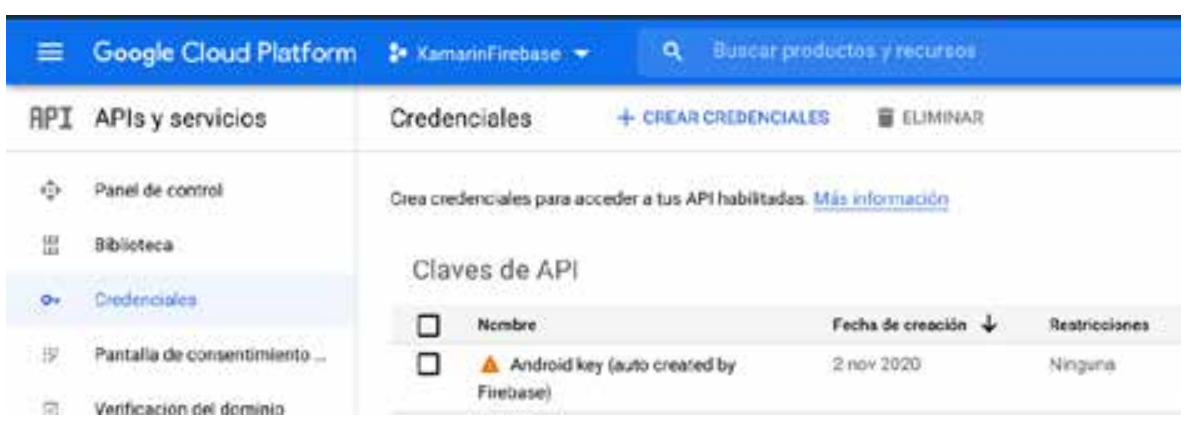

Figura 13. Consola de administración de credenciales de APIs y servicios del API Key creado para la plataforma Android.

Fuente: Fuente: elaboración propia

En las Figuras 14, 15, 16, 17 y 18 se puede ver la creación del proyecto en la plataforma de Facebook for Developers, para la aplicación móvil que va a permitir utilizar el inicio de sesión por medio de esta red social.

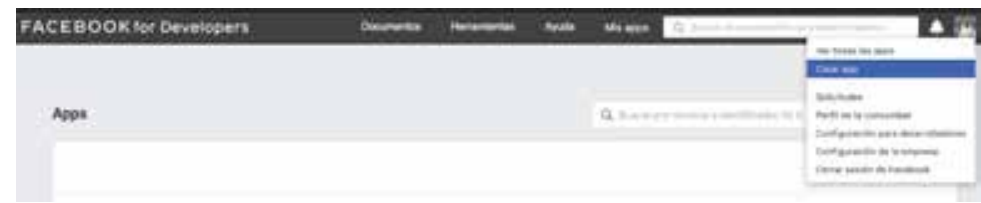

Figura 14. Creación de proyecto en Facebook for Developer.

Fuente: elaboración propia

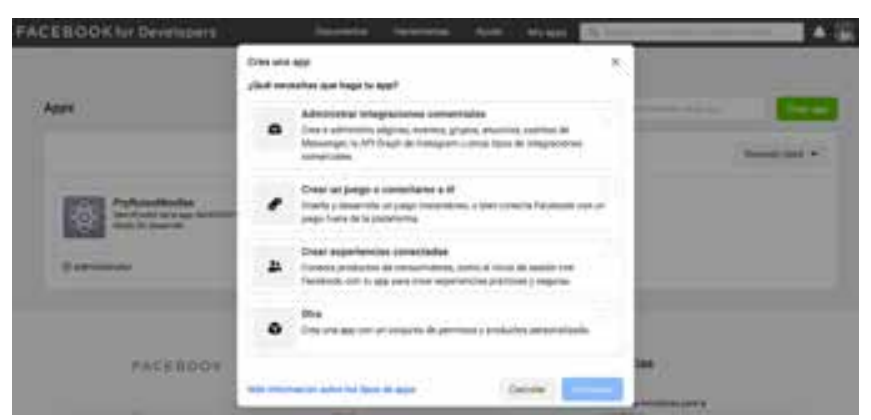

Figura 15. Configuración inicial de proyecto en Facebook for Developer.

Fuente: elaboración propia 


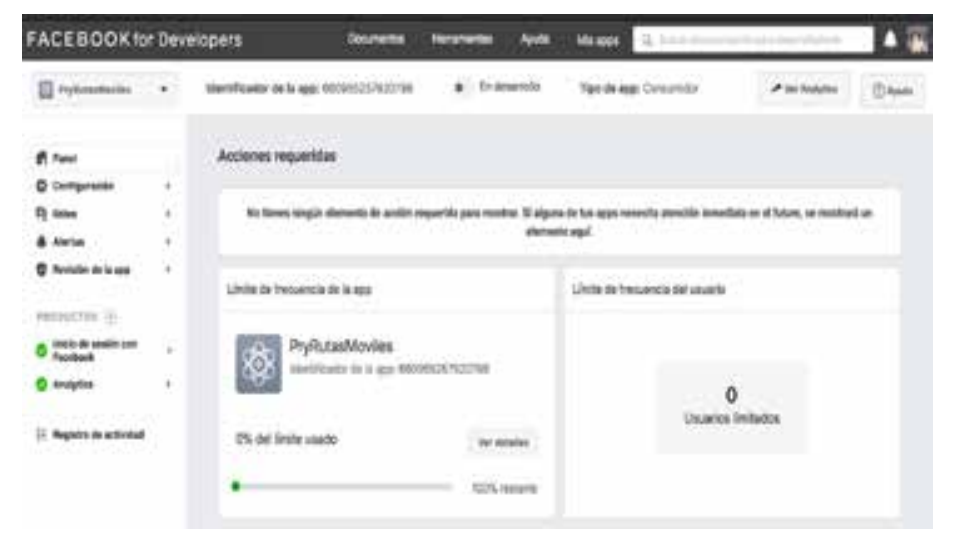

Figura 16. Panel de administración de proyecto en Facebook for Developer.

Fuente: elaboración propia

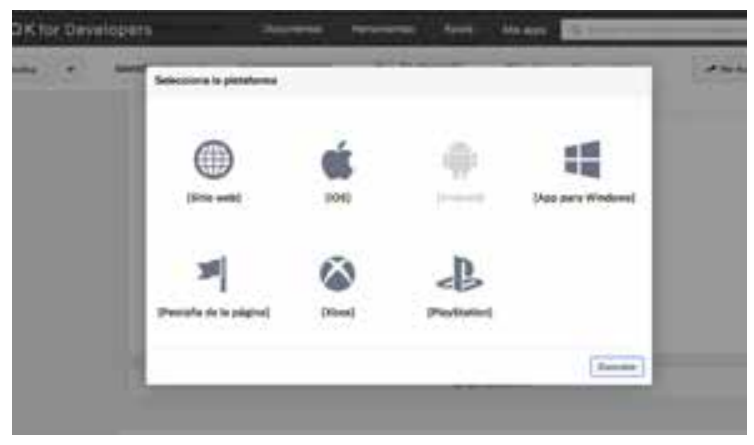

Figura 17. Adición de la plataforma Android en Facebook for Developer.

Fuente: elaboración propia

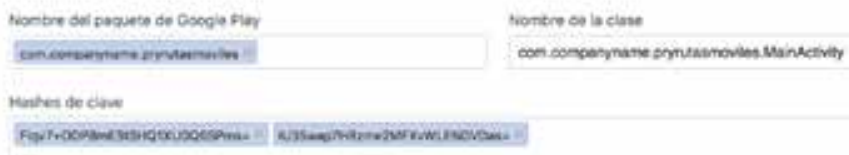

Figura 18. Nombre del paquete Android configurado en el proyecto de Facebook.

Fuente: elaboración propia

En el proyecto de Xamarin, para que todas estas APIs funcionen correctamente requieren de la instalación de los siguientes paquetes:

- FirebaseDatabase.net

- Xamarin.Forms.Maps

- Plugin.FacebookClient

- Xamarin.GooglePlayServices.Auth 
En las Figuras 19, 21, 21 y 22 se pueden visualizar los diferentes paquetes que se incluyeron en el proyecto de Xamarin, previo a su instalación.

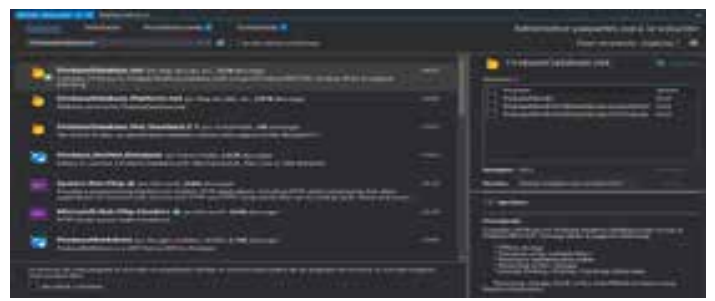

Figura 19. Instalación de Paquete FirebaseDatabase.net Fuente: elaboración propia

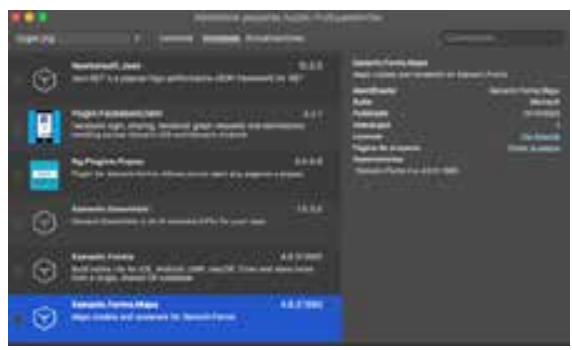

Figura 21. Instalación Paquete Xamarin.Forms.Maps Fuente: elaboración propia

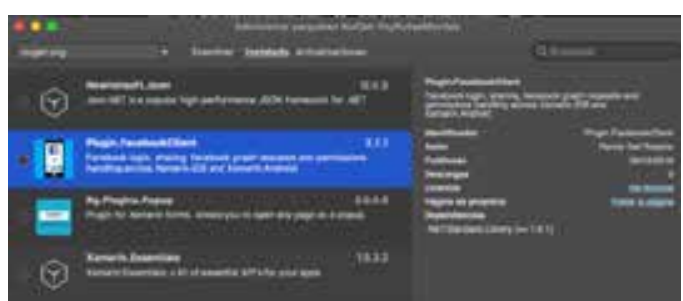

Figura 20. Instalación de Paquete Plugin. FacebookClient Fuente: elaboración propia

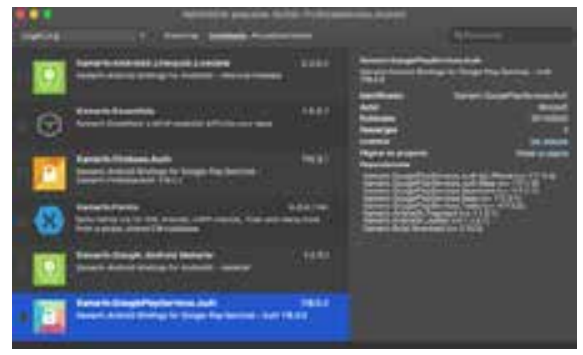

Figura 22. Instalación de Paquete Xamarin. GooglePlayService.Auth

Fuente: elaboración propia

Al finalizar su respectiva instalación y configuración dentro del proyecto se procede a resolver posibles errores y empezar con la codificación del proyecto.

\section{Resultados}

El resultado principal de este trabajo es una aplicación informática para dispositivos móviles, misma que fue validada mediante diferentes pruebas, como:

\subsection{Pruebas funcionales}

En el presente proyecto se realizaron pruebas funcionales de la aplicación móvil con el fin de cumplir los requisitos propuestos y validar que la aplicación funcione correctamente.

En la Figura 23 se puede observar la pantalla de inicio de la aplicación donde el usuario podrá acceder al aplicativo móvil por medio de sus credenciales o usando la autenticación de Google o Facebook. 


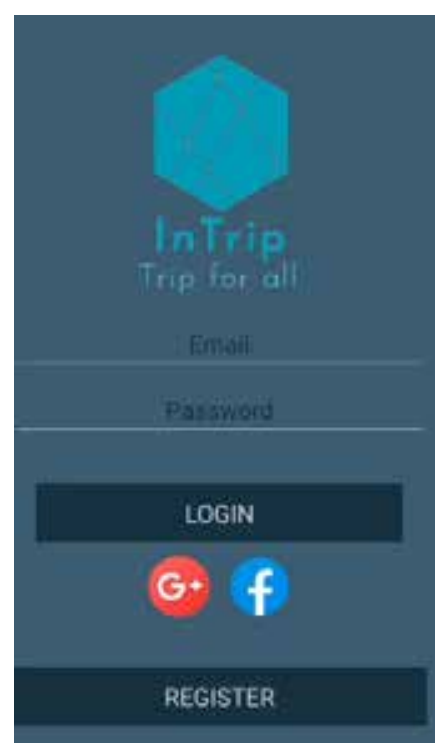

Figura 23. Pantallas de inicio.

Fuente: elaboración propia

En las Figuras 24 y 25 se ve la pantalla de registro de usuarios tanto para pasajeros, como para conductores, estos ingresan su información personal, adicionalmente únicamente los conductores ingresan la información de su vehículo, para ambos tipos de usuarios se utiliza un correo electrónico y una contraseña como credenciales de ingreso. 


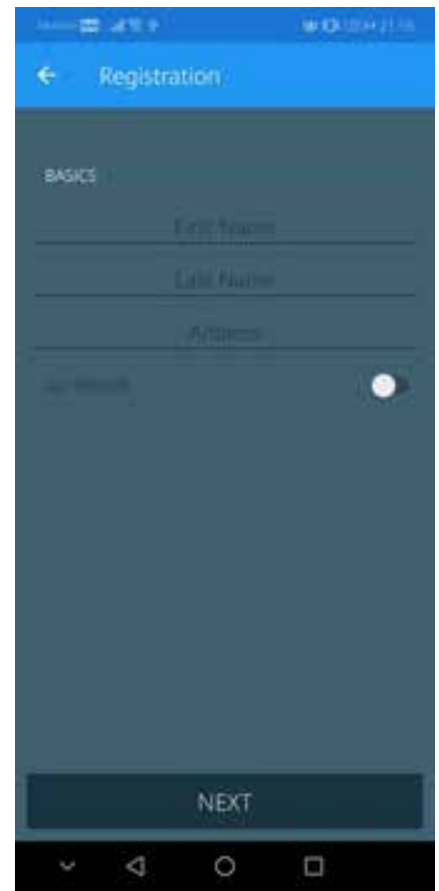

Figura 24. Pantallas de registro para datos personales.

Fuente: elaboración propia

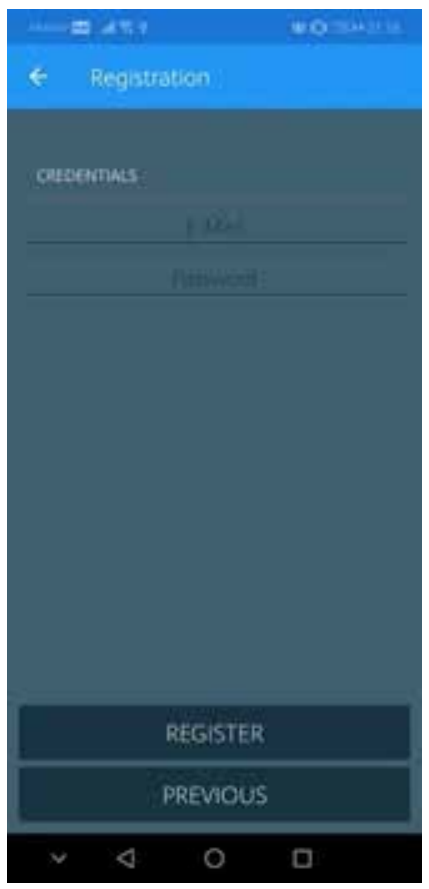

Figura 25. Pantallas de registro para credenciales.

Fuente: elaboración propia

La primera parte de pruebas funcionales fueron realizadas a estas primeras pantallas, lo que permitió validar el registro de usuarios y su ingreso a la aplicación utilizando credenciales o autenticación por redes sociales. Esto permitió corregir errores y posibles problemas con los flujos de funcionamiento.

\subsection{Pantallas de conductor}

En las Figuras 26, 27 y 28 se visualizan las pantallas que tendrá el conductor al iniciar sesión en la aplicación. El conductor podrá realizar: la oferta de su ruta a los pasajeros, ver su perfil de usuario con su información personal y un historial de viajes realizados. 


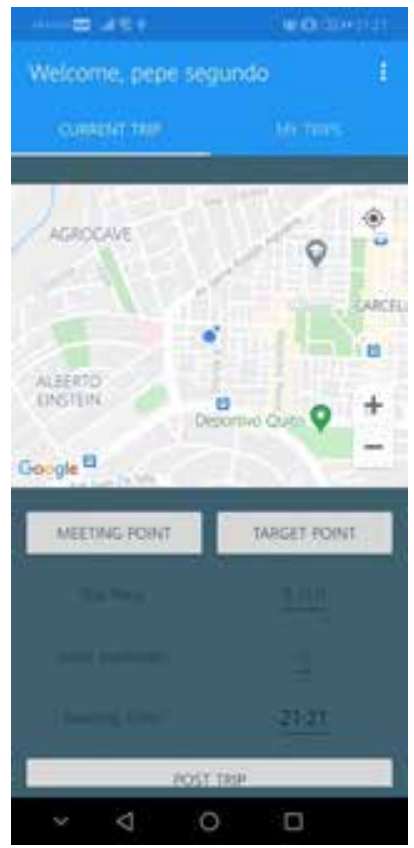

Figura 26. Pantalla de postulación de viaje. Fuente: elaboración propia

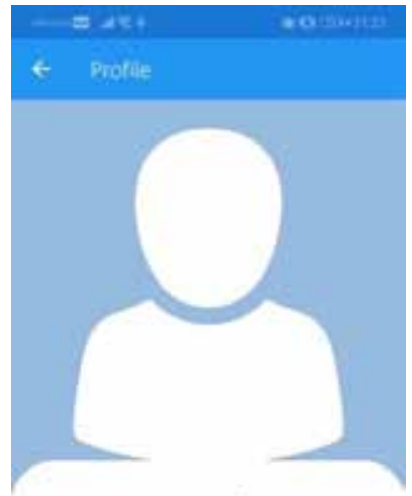

pepe segundo

tmat provilounticion

na tind 2016 myenos

$\checkmark \quad 0 \quad 0$

Figura 27. Pantalla de perfil.

Fuente: elaboración propia

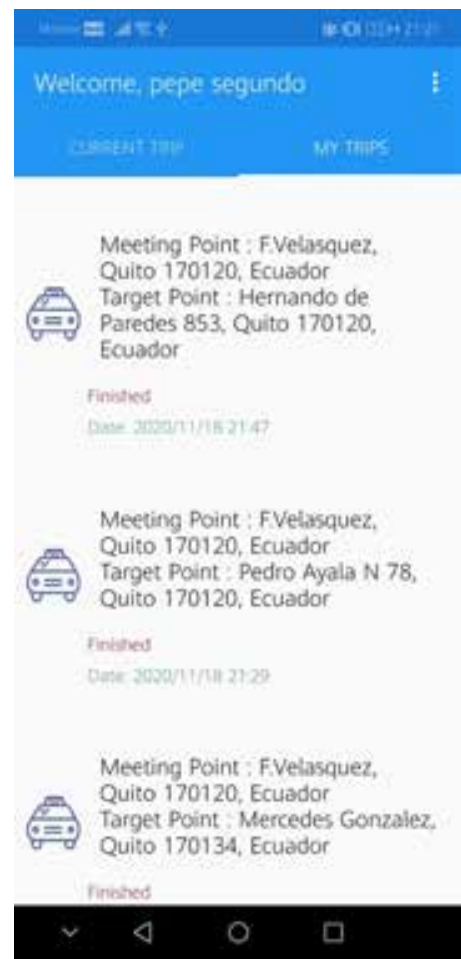

Figura 28. Pantalla de historial de viajes.

Fuente: elaboración propia 
En las Figuras 29 y 30 corresponden a la pantalla del viaje ofertado por el conductor y se mantiene a la espera de los pasajeros. Se muestra información referente al viaje, así como botones de acción para iniciar o finalizar el viaje.

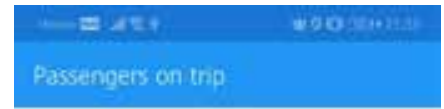

Info Trip

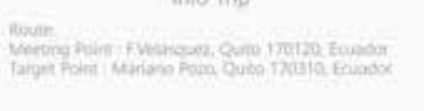

Weeting turie 22:45:00 pior 550

A hency ropas

Passengert

Fow

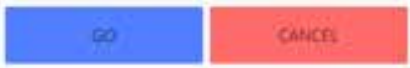

$\checkmark \quad 4 \quad 0 \quad$ a

Figura 29. Pantalla de viaje postulado sin iniciar.

Fuente: elaboración propia

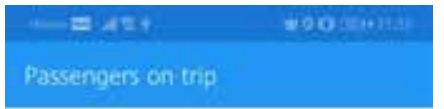

Info Trip

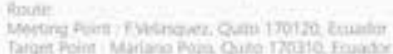

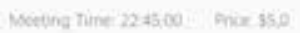

A henty esciat

Pavenger

FBoSH
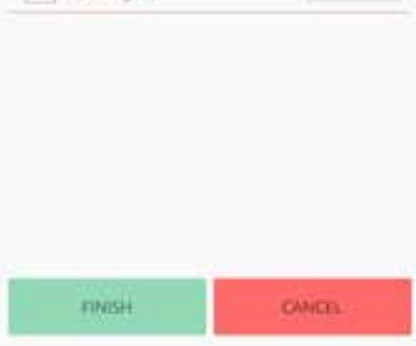

$\checkmark \quad 4 \quad 0 \quad \square$

Figura 30. Pantalla de viaje postulado iniciado.

Fuente: elaboración propia

\subsection{Pantallas de pasajero}

En las Figuras 31, 32 y 33 se observan las pantallas que tendrá el pasajero al iniciar sesión en la aplicación. El pasajero podrá: visualizar los viajes que ofrecen los conductores, ver su perfil de usuario con su información personal y un historial de viajes realizados. 


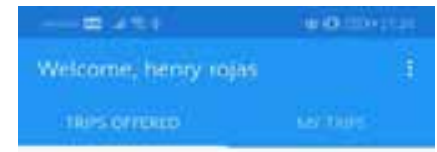

(a)

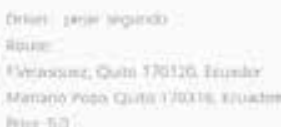

$\checkmark \begin{array}{lll}4 & 0 & \square\end{array}$

Figura 31. Pantalla de viajes ofertados.

Fuente: elaboración propia

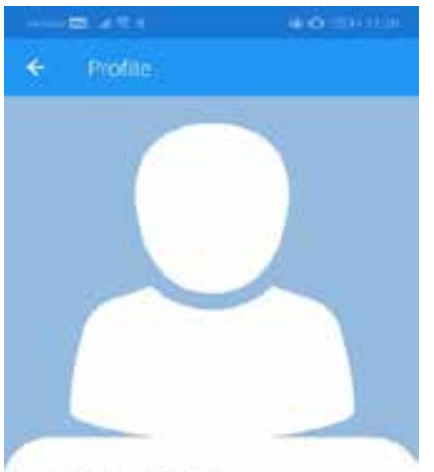

henry rojas

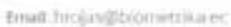

$\vee<\quad$ a

Figura 32. Pantalla de perfil.

Fuente: elaboración propia

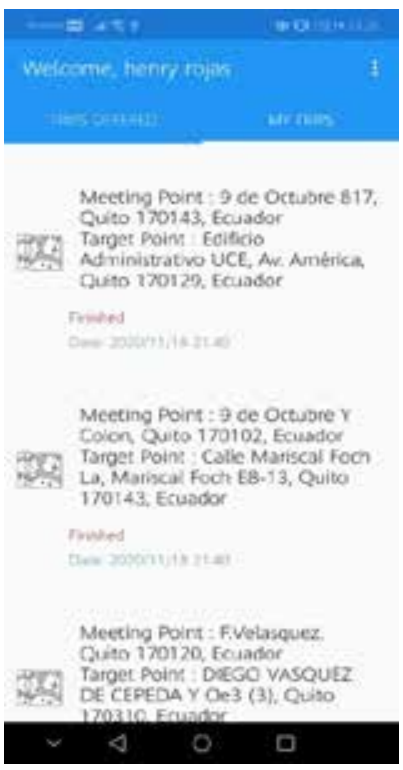

Figura 33. Pantalla de historial de viajes.

Fuente: elaboración propia

En la Figura 34 se muestra la pantalla de visualización del viaje que ofrece un conductor con mayor detalle y decidir si desea o no aceptar dicha oferta. En la Figura 35 se observa la pantalla de un viaje aceptado por el pasajero, esta permanece visible durante el transcurso del viaje hasta que el conductor termine el viaje. 


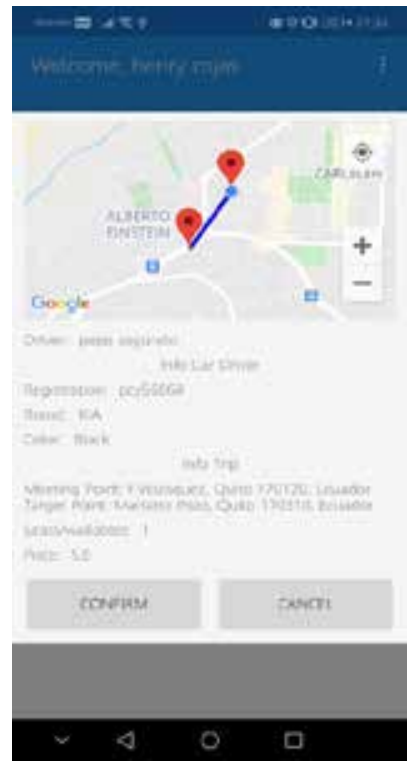

Figura 34. Pantalla detalle del viaje ofertado.

Fuente: elaboración propia
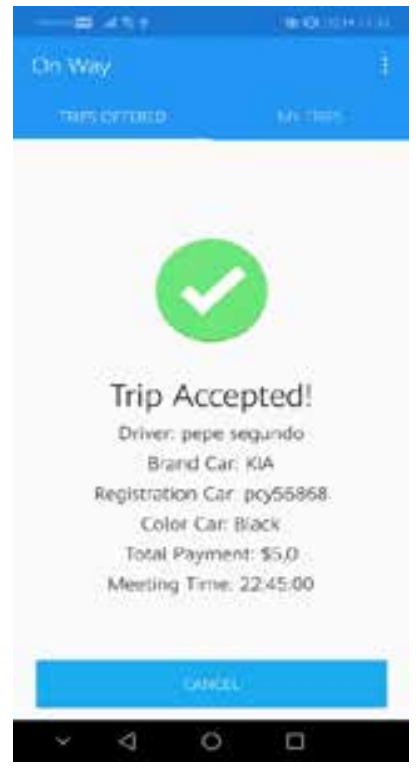

Figura 35. Pantalla de viaje aceptado. Fuente: elaboración propia

La segunda parte de pruebas funcionales fueron realizadas a las pantallas de conductor y pasajero, lo que permitió realizar un flujo completo desde la oferta del viaje hasta su finalización, esto facilitó la corrección de errores y garantizó que la funcionalidad del aplicativo es la adecuada para los usuarios.

\section{Conclusiones}

Con el desarrollo de este proyecto, que tuvo como finalidad ayudar a mejorar el sistema de movilización. InTrip permite ofrecer a conductores la oportunidad de brindar una ruta de viaje con su vehículo, apoyando así a su economía y a pasajeros a llegar a sus destinos mucho más rápido, y por un precio más accesible.

La utilización de la metodología Scrum ayudó a que se administren correctamente los tiempos para cada iteración del proyecto y de existir inquietudes se las resuelva fácilmente. Esto también fue apoyado por el compromiso del equipo de trabajo en el desarrollo del trabajo.

Como parte de las conclusiones, tenemos que la aplicación de la plataforma Firebase que ofrece Google resultó como una herramienta muy útil que dispone de un excelente manejo de datos en tiempo real, reduciendo significativamente el tiempo de desarrollo, esfuerzo en la construcción y despliegue de APIs para el manejo de datos.

En el ámbito económico, el uso de Firebase resulta una buena opción para aplicaciones de baja y media transaccionalidad, ya que resulta ser bastante conveniente tomando en cuenta todo el esfuerzo, tiempo y dinero que implica levantar una estructura propia. 
A su vez, la utilización de mapas de Google permite ofrecer al usuario una mejor experiencia visual sobre la ruta ofrecida por los conductores, lo cual es un recurso muy importante en aplicaciones de movilidad.

Además que, se evidenció que utilizar Xamarin como herramienta de desarrollo móvil ofrece la posibilidad de crear aplicaciones para las dos plataformas móviles más usadas: Android y IOS, lo que permitió ahorrar tiempo y esfuerzo que se pierde al desarrollar en sus herramientas nativas. 


\section{Referencias}

Burbano Ulloa, E. A., Constante Molina, M. A., Hidalgo Guamán, L. M., \& Moya Chiluiza, F. A. (2020). Prototipo móvil para la geolocalización de mascotas callejeras. REVISTA ODIGOS, 1(3), 77-96. https://doi.org/10.35290/ro.v1n3.2020.372

Cabify. (n.d.). Retrieved November 26, 2020, from https://cabify.com/ec

Herrera, X. E. (2020). YACHANA REVISTA CIENTÍFICA. YACHANA Revista Científica, 9(1), 57-67. http://revistas.ulvr.edu.ec/index.php/yachana/article/view/623/361

IDE de Visual Studio, editor de código, Azure DevOps y App Center - Visual Studio. (n.d.). Retrieved November 26, 2020, from https://visualstudio.microsoft.com/es/

inDriver. (n.d.). Retrieved November 26, 2020, from https://indriver.com/es/city

Lage Junior, M., \& Godinho Filho, M. (2010). Variations of the kanban system: Literature review and classification. International Journal of Production Economics, 125(1), 13-21. https://ideas.repec. org/a/eee/proeco/v125y2010i1p13-21.html

Martinez, E. (2001). La evolución de la telefonía móvil. Revista RES, 1, 1-6.

Reyes Rivera, A. J., Vallejos Cango, M. de L., \& Quintana García, D. A. (2020). Nueva realidad: compras en línea y a domicilio. REVISTA ODIGOS, 1(3), 47-60. https://doi.org/10.35290/ ro.v1n3.2020.370

SCRUM - Proyectos Ágiles. (n.d.). Retrieved November 26, 2020, from https://proyectosagiles.org/ que-es-scrum/

Sitio oficial de Uber. (n.d.). Retrieved November 26, 2020, from https://www.uber.com/global/es/ sign-in/

Toasa, R., Silva, C., Silva, C., Goncalves, D., Neves, L., \& Marcelino, L. (2017). Energy consumption behaviour characterization with mobile gamification. Iberian Conference on Information Systems and Technologies, CISTI. https://doi.org/10.23919/CISTI.2017.7975900

Torres, A. G., Quevedo, J. M., García, S. G., \& de la Rosa, C. S. J. (2020). El coronavirus en el ecosistema de los dispositivos móviles: creadores, discursos y recepción - Dialnet. Revista Latina de Comunicación Social. https://dialnet.unirioja.es/servlet/articulo?codigo=7625679

Weller, J. (2020). La pandemia del COVID-19 y su efecto en las tendencias de los mercados laborales. CEPAL. www.cepal.org/apps

Xamarin I Open-source mobile app platform for .NET. (n.d.). Retrieved November 26, 2020, from https://dotnet.microsoft.com/apps/xamarin 